$1 \mathrm{P} 319$

\section{DNA の相互作用に基づいた能動的自己組織化の制御}

Control of Active Self-organization of microtubule by using DNA based interaction

Kyohei Uenishi ${ }^{1}$, Shoki Wada $^{1}$, Daisuke Inoue ${ }^{1}$, Kazuki Sada ${ }^{1,2}$, Akira Kakugo ${ }^{1,2}\left({ }^{1}\right.$ Grad. Sch. of Chem. Sci. and Eng., Hokkaido Univ., ${ }^{2}$ Fac. of Sci. Hokkaido Univ.)

Biomolecular motor such as kinesin converts chemical energy into mechanical work with a high efficiency and moves along the microtubule (MT). MTs, introducing biotin-streptavidin interaction, were integrated into assembled structures on a kinesin-coated surface and MT assemblies provide specific motion. However, reversible control of the MT association / dissociation was not achieved. Hence, we prepared DNAmodified MT and control the self-organization of MTs by using DNA interaction. DNA-modified MTs can reversibly associate and dissociate depending on the DNA sequence used. It will offer means to control the assembly of MT and thereby may widen the application of biomolecular motor based nanodevice.

Cell trapping device for observation of connexin function by a single cell device

Kosuke Inoue $^{1,2}$, Koki Kamiya ${ }^{1,4}$, Yuta Abe ${ }^{1,2}$, Toshihisa Osaki ${ }^{1,3}$, Norihisa Miki $^{2}$, Shoji Takeuchi ${ }^{1,3}$ ( ${ }^{1}$ Kanagawa Academy of Science and Technology, ${ }^{2}$ Keio University, ${ }^{3}$ Institute of Industrial Science, the University of Tokyo, ${ }^{4}$ PRESTO, Japan Science and Technology Agency)

Membrane proteins play an important role in several biological functions including single transduction, energy production and cellular communication. Understanding the characteristic of membrane proteins is important for drug screening. Accordingly, the cells should be trapped for the analysis of membrane proteins' functions. In this study, we first arranged three cells in the order of donor-acceptor-acceptor cells using the parylene slide device in which the number and position of trapping cells was controlled by sliding parylene rails $(5 \mu \mathrm{m} \times 54 \mu \mathrm{m})$ on comb-shaped blocker. Next, we investigated the permeability of two fluorescent molecular (calcein and cytored) in $\mathrm{Cx}$ channel from the donor cell to the acceptor cells on the parylene rail.

1P321

\section{質量を変えたレプリカ交換分子動カ学法}

Mass-scaling replica-exchange molecular dynamics method

Tetsuro Nagai, Takuya Takahashi (College of Life Sciences, Ritsumeikan University)

We present a new method of replica-exchange molecular dynamics (REMD) simulation, mass-scaling REMD (MSREMD) method, which improves trajectory accuracy at high temperatures and thereby contributes to numerical stability. In addition, the MSREMD method can simplify a replica-exchange routine by eliminating velocity scaling. As a pilot system, a Lennard-Jones fluid is simulated. The results show that the MSREMD method improves the trajectory accuracy at high temperatures over the conventional REMD method. We compare the computational costs of the REMD and MSREMD simulations, concluding that the MSREMD method optimizes the computational resources with simpler algorithm under the constant trajectory accuracy at all temperatures.
Takeshi Sugawara ${ }^{1,2}$, Shota Masuda ${ }^{3}$, Jun-ichi Uewaki ${ }^{1,2}$, Akinori Awazu ${ }^{1,2}$, Hiraku Nishimori ${ }^{1,2}$, Masaru Ueno ${ }^{1,3}\left({ }^{1} R c M c D\right.$, Hiroshima Univ., ${ }^{2}$ Department of Mathematical and Life Sciences, Faculty of Science, Hiroshima University, ${ }^{3}$ Department of Molecular Biotechnology, Graduate School of Advanced Sciences of Matter, Hiroshima University)

Based on genome-wide chromatin interactions obtained from 3C experiments, 3D models of chromosomes have been constructed for some organisms. However, such models are constructed from population-based data on fixed cells and thus do not contain information about dynamic aspects of chromosomes. We are interested in how chromatin dynamically changes its structure during cell cycle, then we visualized gene loci in $S$. pombe and tracked the 3D positions of the loci. We statistically analyzed the positions and dynamics of the loci, and then revealed quantitative features of chromatin dynamics. In this poster presentation, we will report recent results and finally discuss our perspectives toward understanding possible roles that chromatin dynamics play within the cell nucleus.

Dynamical heterogeneity and dynamics of cage breaking and formation in colloidal fluids

Preetom Nag ${ }^{1,2}$, Hiroshi Teramoto ${ }^{1,2}$, Chun-Biu $\mathrm{Li}^{2,3,4}$, Tamiki Komatsuzaki ${ }^{1,2,4}\left({ }^{1}\right.$ Graduate School of Life Science, Transdisciplinary Life Science Course, Hokkaido University, ${ }^{2}$ Molecule and Life Nonlinear Sciences Laboratory, Research Institute for Electronic Science, Hokkaido University, ${ }^{3}$ Graduate School of Science, Department of Mathematics, Hokkaido University, ${ }^{4}$ Research Center for Integrative Mathematics, Hokkaido University)

We study on colloidal fluids to understand system's mechanical response under a microscopic perturbation arises from a single optically trapped particle. Particles that undergo distortions of their packing configuration by the time scale of perturbation, resulting in a cage breaking and formation dynamics, are investigated by the power in Wavelet transform. We compare such a cage breaking and fomation dynamics with the underlying dynamical structure identified as finite-time Lyapunov exponent (FTLE) based Lagrangian Coherent Structures (LCSs) which are mobile separatrices that separate the flow into regions of different dynamical behavior. We have established a relationship between LCSs and cage breaking and formation dynamics. 